\title{
Cardiomyocyte mitochondria as targets of humoral factors released by remote ischemic preconditioning
}

\author{
Nilguen Gedik ${ }^{1}$, Leonardo Maciel ${ }^{1,2}$, Christiane Schulte ${ }^{1}$, Andreas Skyschally ${ }^{1}$, Gerd Heusch ${ }^{1}$, \\ Petra Kleinbongard ${ }^{1}$
}

\begin{abstract}
${ }^{1}$ Institute for Pathophysiology, West German Heart and Vascular Centre Essen, University of Essen, Medical School, Essen, Germany

2Laboratory of Cardiac Electrophysiology, Institute of Biophysics Carlos Chagas Filho, Federal University of Rio de Janeiro, Rio de Janeiro, RJ, Brazil
\end{abstract}

Submitted: 13 June 2016

Accepted: 30 June 2016

Arch Med Sci 2017; 13, 2: 448-458

DOI: https://doi.org/10.5114/aoms.2016.61789

Copyright $\odot 2016$ Termedia \& Banach

\section{Abstract}

Introduction: Remote ischemic preconditioning (RIPC) reduces myocardial infarct size, and protection can be transferred with plasma to other individuals, even across species. Mitochondria are the end-effectors of cardioprotection by local ischemic conditioning maneuvers. We have now analyzed mitochondrial function in response to RIPC.

Material and methods: Plasma from pigs undergoing placebo or RIPC (infarct size reduction by $67 \%$ in RIPC pigs compared to placebo) was transferred to isolated perfused rat hearts subjected to 30 min global ischemia followed by 120 min reperfusion for infarct size measurement. Additional experiments were terminated at $10 \mathrm{~min}$ reperfusion to isolate mitochondria for functional measurements. Effects of RIPC pig plasma were compared to local ischemic preconditioning (IPC) or to infusion of tumor necrosis factor $\alpha$ (TNF- $\alpha$ ).

Results: Ischemia/reperfusion (I/R) induced an infarct of $41 \pm 2 \%$ of total ventricular mass. Placebo pig plasma did not affect infarct size (38 \pm 1 , $p=0.13)$. The RIPC pig plasma reduced infarct size $(27 \pm 2, p<0.001)$, as did IPC $(20 \pm 1, p<0.001)$ and TNF- $\alpha(28 \pm 2, p<0.001)$. Associated with cardioprotection, reductions of mitochondrial adenosine diphosphate (ADP) -stimulated respiration, adenosine triphosphate (ATP) production and calcium retention capacity $(C R C)$ by I/R and placebo pig plasma were prevented by RIPC pig plasma, as they were by IPC and TNF- $\alpha$. Mitochondrial reactive oxygen species production ( $\mathrm{nmol} \mathrm{H}_{2} \mathrm{O}_{2} / 100 \mu \mathrm{g}$ protein) induced by I/R $(272 \pm 34)$ was comparable in response to placebo pig plasma $(234 \pm 28$, $p=0.37)$ and was reduced by RIPC pig plasma $(83 \pm 15, p<0.001)$ as well as by IPC $(78 \pm 21, p<0.001)$ and TNF- $\alpha(125 \pm 42, p=0.002)$.

Conclusions: In rat myocardium, mitochondria are an intracellular target of protection induced by humoral factors retrieved from pigs undergoing RIPC.

Key words: cardioprotection, humoral factor, mitochondria, remote ischemic preconditioning.

\section{Introduction}

Repetitive brief episodes of myocardial ischemia/reperfusion (I/R) before sustained myocardial ischemia and subsequent reperfusion, i.e. local ischemic preconditioning (IPC), protect the myocardium from irreversible I/R injury [1]. Ischemic preconditioning in an organ remote from the heart (RIPC) confers similar cardioprotection. The protection by RIPC has been

\author{
Corresponding author: \\ PD Dr. rer. nat. Petra \\ Kleinbongard \\ Institut für Pathophysiologie \\ Westdeutsches Herz- \\ und Gefäßzentrum Essen \\ Universitätsklinikum Essen \\ Hufelandstr. 55 \\ 45122 Essen, Germany \\ Phone: +49-201-723-2763 \\ Fax: +49-201-723-4481 \\ E-mail: petra.kleinbongard@ \\ uk-essen.de
}


confirmed experimentally by infarct size reduction in all animal species tested so far [2]. Likewise, RIPC by repetitive limb I/R attenuates myocardial injury in patients undergoing elective interventional [3] or surgical coronary revascularization [4-6], and also in patients with acute myocardial infarction [7-10]. The protection was confirmed by reduced cardiac biomarker release [3-6], by cardiac imaging $[7,8,10]$, and by improved short- $[6,8]$ and longterm clinical outcome $[3,5,11]$. However, in two recent large-scale randomized trials, ERICCA and RIPHeart, RIPC neither reduced biomarker release nor improved clinical outcome of patients undergoing cardiac surgery $[12,13]$. To improve the effective use of RIPC in patients, a better understanding of how the remote stimulus is transferred from the ischemic/reperfused organ or limb to the heart, as well as identification of its intracellular target in the protected organ, is needed $[14,15]$.

The cardioprotective signal from the ischemic/ reperfused organ or limb to the heart is transferable with plasma [16-18] or a plasma-derived dialysate/filtrate $[19,20]$ from one individual to another, even across species. Investigations deciphering possible myocardial intracellular targets of RIPC were inspired by studies on local IPC or postconditioning. Mitochondria are viewed as end-effectors of these cardioprotective strategies. The preservation of mitochondrial function after $I / R$ is decisive for survival of cardiomyocytes and thus salvage of the myocardium [21, 22]. Whether mitochondria are also the target of cardioprotection by RIPC is still under investigation. In rats, myocardial infarct size reduction by RIPC is associated with preserved mitochondrial morphology [23], maintained mitochondrial membrane potential and increased mitochondrial manganese superoxide dismutase (MnSOD) content [24]. Several studies have also reported changes in mitochondrial function by RIPC: mitochondrial respiration was improved in rat hearts [25] and in right atrial appendages in situ of patients undergoing cardiac surgery [26, 27]. Transfer of rabbit RIPC plasma dialysate to isolated perfused neonatal rabbit hearts also improved mitochondrial respiration but had no effect on mitochondrial permeability transition pore (mPTP) opening [28]. However, none of these latter studies demonstrated an association of improved mitochondrial function with infarct size reduction.

The available data can therefore not clarify a relationship between improved mitochondrial function and cardioprotection, i.e. infarct size reduction by RIPC. Whether or not mPTP opening, which is decisive for cardiomyocyte survival or death at early reperfusion [29-31], is associated with infarct size reduction by RIPC is also not clear at this point. Therefore, in this study we used an established setup with humoral across-species transfer of RIPC's cardioprotection from an anesthetized pig model in situ to an in vitro isolated perfused rat heart model subjected to global I/R [18]. Mitochondria were isolated from the isolated perfused rat hearts at early reperfusion. Mitochondrial function, i.e. mitochondrial adenosine diphosphate (ADP)-stimulated respiration, calcium retention capacity (CRC) to estimate MPTP opening, adenosine triphosphate (ATP) production and reactive oxygen species (ROS) production, was measured at physiological temperature of $37^{\circ} \mathrm{C}$. For comparison of humorally transferred RIPC cardioprotection, isolated perfused rat hearts were also subjected to local IPC and to infusion of tumor necrosis factor $\alpha$ (TNF- $\alpha$ ), which is known to be an endogenous humoral factor of cardioprotection [32-34].

\section{Material and methods}

\section{Materials}

Chemicals were of the highest quality available, and all solutions were freshly prepared using Milli-Q water or high quality analytical grade organic solvents and, where appropriate, sterilized prior to use. Materials were obtained from Sigma-Aldrich (Deisenhofen, Germany) or purchased as indicated.

\section{Plasma separation after RIPC or placebo maneuver in pigs}

Anesthetized pigs were subjected to RIPC $(4 \times 5 \mathrm{~min} / 5 \mathrm{~min}$ hindlimb I/R, $n=10)$ or placebo $(n=10)$ before $60 \mathrm{~min} / 180 \mathrm{~min}$ coronary occlusion/reperfusion. One hour after the RIPC or placebo maneuver, 100 to $120 \mathrm{ml}$ of arterial blood was withdrawn and sampled in vials containing lithium-heparin and immediately centrifuged at $800 \mathrm{~g}$ and $4^{\circ} \mathrm{C}$ for $10 \mathrm{~min}$. Separated plasma was centrifuged at $4500 \mathrm{~g}$ and $4^{\circ} \mathrm{C}$ for an additional $10 \mathrm{~min}$. The separated plasma was stored at $-80^{\circ} \mathrm{C}$ for later use and again centrifuged for $10 \mathrm{~min}$ at $4500 \mathrm{~g}$ and filtered ( $0.2 \mu \mathrm{m}$ pore size) before use. For details see Skyschally et al. [18].

\section{Experiments in isolated rat hearts}

The experimental protocols were approved by the Landesamt für Natur, Umwelt und Verbraucherschutz Nordrhein-Westfalen, Germany (B1322/12), and the investigation conforms to the Guide for the Care and Use of Laboratory Animals published by NIH Publication 85-23, revised 1996 [35].

Male Lewis rats (age: 10-14 weeks, weight: 250$400 \mathrm{~g})$ were anesthetized by intraperitoneal injection of sodium pentobarbital (400 mg/kg; Narcoren, Merial, Hallbergmoos, Germany). The injection was supplemented with $1000 \mathrm{IU}$ of heparin to attenuate coagulation. The hearts were rapidly excised and placed in ice-cold saline before they were mounted 
on a Langendorff apparatus. Modified Krebs-Henseleit buffer (KHB; in mmol/l: $\mathrm{NaCl} 118.0, \mathrm{KCl} 4.7$, $\mathrm{MgSO}_{4}$ 1.6, $\mathrm{KH}_{2} \mathrm{PO}_{4} 1.2$, glucose 5.6, $\mathrm{NaHCO}_{3} 24.9$, sodium pyruvate $2.0, \mathrm{CaCl}_{2} 2.0$; gassed with $95 \% \mathrm{O}_{2}$ and $5 \% \mathrm{CO}_{2}$ in a $37^{\circ} \mathrm{C}$ pre-warmed reservoir) was used for retrograde perfusion at constant pressure (65-68 $\mathrm{mm} \mathrm{Hg}$ ). The perfusate temperature was held constant by a heat exchanger located next to the aortic cannula. A fluid-filled latex balloon was inserted through the left atrium into the left ventricle and connected to a pressure transducer (CODAN pvb Medical, Lensahn, Germany). Minimal left ventricular pressure $\left(\mathrm{LVP}_{\min }\right)$ was set to $5-15 \mathrm{~mm} \mathrm{Hg}$ by balloon inflation to control ventricular preload, and maximal systolic left ventricular pressure $\left(\right.$ LVP $\left._{\max }\right)$ was measured. Heart rate was set to $400 \mathrm{bpm}$ by atrial pacing. Coronary flow (CF) was measured by an inline ultrasonic transit time flow probe (ME2PXN Transonic, Maastricht, NL) connected to the aortic cannula. During the whole experiment, the hearts were continuously immersed in $37^{\circ} \mathrm{C}$ warm buffer to avoid hypothermia. Hearts were allowed to stabilize for 20 min before a protocol was started (the last $30 \mathrm{~s}$ were defined as baseline).

\section{Experimental protocols}

Sham: Hearts were perfused with KHB solution for 60 or $170 \mathrm{~min}$.

I/R: Global ischemia was induced for 30 min by full stop of retrograde perfusion followed by 10 or 120 min reperfusion.

Placebo/RIPC pig plasma: Infarct size of pig hearts was reduced with RIPC (12 $\pm 2 \%$ of area at risk) in comparison to placebo $(36 \pm 6 \%$; $p<0.05)$ and was comparable with the size measured in our prior study [18]. Pig plasma was infused for $8 \mathrm{~min}$ via a syringe pump into the KHB solution (1:10 volume ratio) before passing the heat exchanger. After $2 \mathrm{~min}$ washout of the plasma with KHB solution to avoid adherence of proteins from stagnant plasma to the Langendorff apparatus, global ischemia was induced as in the I/R protocol, and plasma infusion was continued during the initial 5 min of reperfusion.

Local IPC: Three cycles of $3 \mathrm{~min} / 3 \mathrm{~min}$ of I/R were induced immediately before induction of the I/R protocol.

TNF- $\alpha$ infusion: TNF- $\alpha(0.5 \mathrm{ng} / \mathrm{ml})$ was perfused for 7 min via a syringe pump into the KHB solution before passing the heat exchanger. A washout with KHB solution for 10 min was performed before induction of the I/R protocol to activate the cardioprotection by TNF- $\alpha$ [36].

For infarct size measurement experimental protocols were terminated after 120 min reperfusion. For measurement of mitochondrial function, experimental protocols were terminated after 10 min reperfusion to isolate mitochondria from the rat hearts.

\section{Coronary flow and left ventricular developed pressure}

Coronary flow and left ventricular developed pressure $\left(\mathrm{LVDP}=\mathrm{LVP}_{\max }-\mathrm{LVP}_{\min }\right)$ were analyzed at the following time points of the protocols: at baseline, after plasma infusion/IPC/TNF- $\alpha$ infusion (intervention), at 5/25 min ischemia, and at $10 / 30$ min reperfusion. Mean values over $30 \mathrm{~s}$ at the respective time points were calculated.

\section{Infarct size measurement}

After 120 min reperfusion hearts were frozen in Cryomatrix (Thermo Scientific, Waltham, MA, USA) at $-20^{\circ} \mathrm{C}$ and cut into transverse $1 \mathrm{~mm}$ thick slices. Infarcted tissue was demarcated by staining with $2 \%$ triphenyl tetrazolium chloride (TTC) solution containing $5 \%$ dextran at $37^{\circ} \mathrm{C}$ for $20 \mathrm{~min}$. Stained slices were weighed and photographed from both sides. The total slice area and the infarcted area were measured by computer-assisted planimetry. After normalization for weight, infarct size was calculated as the fraction of total ventricular mass [18].

\section{Mitochondria isolation and measurement of mitochondrial function}

All procedures were performed on ice or at $4^{\circ} \mathrm{C}$. After 10 min reperfusion isolated rat hearts were rapidly removed from the Langendorff apparatus, placed in ice-cold isolation buffer $(\mathrm{pH} 7.4)$ containing (in mmol/l) 250 sucrose, 10 HEPES, 1 ethylene glycol tetraacetic acid (EGTA), with $0.5 \% \mathrm{w} / \mathrm{v}$ bovine serum albumin (BSA), minced thoroughly using scissors, and then homogenized with a tissue homogenizer (Ultra-Turrax, IKA, Staufen, Germany) using two $10 \mathrm{~s}$ treatments at a shaft rotation rate of $6,500 \mathrm{rpm}$ to release the subsarcolemmal mitochondria. Further homogenization with a Teflon pestle in the presence of proteinase type XXIV (8 IU/mg tissue weight) then released interfibrillar mitochondria. The homogenate containing subsarcolemmal and interfibrillar mitochondria was centrifuged at $700 \mathrm{~g}$ for $10 \mathrm{~min}$. The supernatant was collected and centrifuged at 14,000 g for $10 \mathrm{~min}$. The resulting pellet was resuspended in isolation buffer without BSA and centrifuged at 10,000 g for $5 \mathrm{~min}$. The latter procedure was repeated, and the pellet was resuspended in isolation buffer [37]. The protein concentration of the resuspended pellet was determined using a protein assay (Lowry method, Bio-Rad, Hercules, CA, USA) with BSA as standard (Thermo Scientific, Waltham, MA, USA).

\section{Mitochondrial respiration}

Mitochondrial respiration was measured with a Clark-type electrode (Strathkelvin, Glasgow, UK) at $37^{\circ} \mathrm{C}$ during magnetic stirring in incubation 
buffer containing in mmol/l: $125 \mathrm{KCl}, 10 \mathrm{MOPS}$, $2 \mathrm{MgCl}_{2}, 5 \mathrm{KH}_{2} \mathrm{PO}_{4}, 0.2$ EGTA with 5 glutamate and 5 malate as substrates for complex I. The oxygen electrode was calibrated using a solubility coefficient of $217 \mathrm{nmol} \mathrm{O} / \mathrm{ml}$ at $37^{\circ} \mathrm{C}$.

For the measurement of complex I respiration, suspended mitochondria (corresponding to a protein amount of $50 \mu \mathrm{g}$ ) were added to $0.5 \mathrm{ml}$ of incubation buffer. After $2 \mathrm{~min}, 1 \mathrm{mmol} / \mathrm{ADP}$ was added and ADP-stimulated respiration was measured over 2-3 min.

Hereafter, mitochondria were used to either measure complex IV respiration and maximal uncoupled oxygen uptake in the respiration chamber, or incubation buffer containing mitochondria was taken from the respiration chamber to measure ATP production or ROS production, respectively.

Complex IV respiration was stimulated by adding $\mathrm{N}, \mathrm{N}, \mathrm{N}^{\prime}, \mathrm{N}^{\prime}$-tetramethyl-p-phenylenediamine (TMPD, $300 \mu \mathrm{mol} / \mathrm{l})$ and ascorbate $(3 \mathrm{mmol} / \mathrm{l})$, which donates electrons to cytochrome oxidase via the reduction of cytochrome c. Maximal uncoupled oxygen uptake was measured in the presence of $30 \mathrm{nmol} / \mathrm{l}$ carbonyl cyanide-p-trifluoromethoxyphenyl-hydrazone (FCCP) [37].

\section{Mitochondrial ATP production}

After measurement of ADP-stimulated respiration, the incubation buffer containing mitochondria was taken from the respiration chamber and immediately supplemented with ATP assay mix (diluted $1: 5$ ). Mitochondrial ATP production after measurement of respiration was determined immediately and compared with ATP standards using a 96-well white plate and a Cary Eclipse spectrophotometer (Varian, Mulgrave, Victoria, Australia) at $560 \mathrm{~nm}$ emission wavelength [37].

\section{Calcium retention capacity}

Calcium retention capacity was determined using suspended mitochondria (corresponding to a protein amount of $100 \mu \mathrm{g}$ ) in $1 \mathrm{ml}$ of CRC buffer containing (in mmol/l) $125 \mathrm{KCl}, 10 \mathrm{MOPS}$, $2 \mathrm{MgCl}_{2}, 5 \mathrm{KH}_{2} \mathrm{PO}_{4}$ with 5 glutamate and 5 malate as substrates in the presence of ADP $(0.4 \mathrm{mmol} / \mathrm{l})$, at $37^{\circ} \mathrm{C}$. Calcium green-5N (0.5 $\mu \mathrm{mol} / \mathrm{l}$, Life Technologies, Carlsbad, CA, USA) was used to measure the extramitochondrial calcium concentration in a spectrophotometer (Cary Eclipse, Varian, Mulgrave, Victoria, Australia) at $500 \mathrm{~nm}$ emission and $530 \mathrm{~nm}$ extinction wavelengths. Pulses of $5 \mathrm{nmol} \mathrm{CaCl}_{2}$ were added every minute until a rapid increase in calcium green fluorescence indicated MPTP opening [38]. The immunosuppressant cyclosporine A delays mPTP opening by interaction with cyclophilin $D$ to keep the pore closed [39]. Therefore, additional measurements were performed with cyclosporine A $(10 \mu \mathrm{mol} / \mathrm{l})$ as a positive control.

\section{ROS production}

The Amplex Red Hydrogen Peroxide Assay (Life Technologies, Carlsbad, CA, USA) was used to determine the ROS concentration in the extramitochondrial space. Amplex Red reacts in a $1: 1$ stoichiometry with peroxides under catalysis by horseradish peroxidase (HRP) and produces highly fluorescent resorufin. The incubation buffer containing mitochondria was removed from the respiration chamber and immediately supplemented with $50 \mu \mathrm{mol} / \mathrm{l}$ Amplex UltraRed and $2 \mathrm{U} / \mathrm{ml} \mathrm{HRP}$. The supernatant was collected after 120 min of incubation in the dark. The ROS concentration was determined and compared with $\mathrm{H}_{2} \mathrm{O}_{2}$ standards using a 96-well black plate and a Cary Eclipse fluorescence spectrophotometer (Varian, Mulgrave, Victoria, Australia) at $540 \mathrm{~nm}$ emission and $580 \mathrm{~nm}$ extinction wavelengths [37].

\section{Statistical analysis}

Data are presented as mean \pm standard error of the mean (SEM). Coronary flow and LVDP were analyzed by two-way ANOVA for repeated measures. Infarct size, mitochondrial respiration, ATP production, CRC and ROS production were analyzed by one-way ANOVA. When a significant difference was detected, one-way and two-way ANOVA were followed by Fisher's LSD post-hoc tests (SigmaStat 2.03, SPSS Inc., Chicago, IL, USA). Differences were considered significant at the level of $p<0.05$.

\section{Results}

Coronary flow and left ventricular developed pressure in isolated perfused rat hearts

Coronary flow and LVDP were not different between groups at baseline and did not change during the experiments in the rat hearts with sham. After plasma infusion, during ischemia, and during reperfusion, CF and LVDP were comparable between I/R, placebo and RIPC pig plasma, respectively. CF and LVDP of rat hearts with IPC and TNF- $\alpha$ infusion were comparable with I/R during ischemia, but rat hearts recovered better with IPC at reperfusion (Table I).

\section{Infarct size of isolated perfused rat hearts}

Almost no infarction was detectable with sham. Compared to I/R, infarct size was similar with placebo pig plasma, whereas RIPC pig plasma reduced infarct size. Comparable infarct size reduction was observed with local IPC and TNF- $\alpha$ infusion (Figure 1). 
Nilguen Gedik, Leonardo Maciel, Christiane Schulte, Andreas Skyschally, Gerd Heusch, Petra Kleinbongard

Table I. Coronary flow and left ventricular developed pressure of isolated perfused rat hearts

\begin{tabular}{|c|c|c|c|c|c|}
\hline \multirow[t]{2}{*}{ Variable } & \multirow[t]{2}{*}{ Time } & \multicolumn{2}{|c|}{ Rat hearts for infarct size measurement } & \multicolumn{2}{|c|}{ Rat hearts for mitochondria preparation } \\
\hline & & $\begin{array}{c}\mathrm{CF}_{\text {mean }} \\
{[\mathrm{ml} / \mathrm{min}]}\end{array}$ & $\begin{array}{l}\text { LVDP }_{\text {max }} \\
{[\mathrm{mm} \mathrm{Hg]}}\end{array}$ & $\begin{array}{c}\mathrm{CF}_{\text {mean }} \\
{[\mathrm{ml} / \mathrm{min}]}\end{array}$ & $\begin{array}{l}\text { LVDP }_{\text {max }} \\
{[\mathrm{mm} \mathrm{Hg]}}\end{array}$ \\
\hline \multirow{6}{*}{$\begin{array}{l}\text { I/R } \\
(n=8 / 8)\end{array}$} & Baseline & $13.0 \pm 0.6$ & $74.5 \pm 6.7$ & $12.6 \pm 0.9$ & $91.3 \pm 6.0$ \\
\hline & After intervention & $12.4 \pm 0.6$ & $71.6 \pm 7.9$ & $12.6 \pm 0.9$ & $91.9 \pm 6.2$ \\
\hline & Ischemia 5 min & $0.0 \pm 0.0^{*}$ & $0.4 \pm 0.2^{*}$ & $0.0 \pm 0.0^{*}$ & $0.6 \pm 0.3^{*}$ \\
\hline & Ischemia $25 \mathrm{~min}$ & $0.0 \pm 0.0^{*}$ & $0.3 \pm 0.1^{*}$ & $0.0 \pm 0.0^{*}$ & $3.8 \pm 3.6^{*}$ \\
\hline & Reperfusion 10 min & $8.5 \pm 0.8^{*}$ & $22.9 \pm 7.8^{*}$ & $7.9 \pm 1.2^{\star}$ & $25.4 \pm 6.6^{\star}$ \\
\hline & Reperfusion 30 min & $7.7 \pm 0.9^{*}$ & $31.0 \pm 7.6^{*}$ & - & - \\
\hline \multirow{6}{*}{$\begin{array}{l}\text { pPlacebo } \\
(n=10 / 10)\end{array}$} & Baseline & $12.3 \pm 0.4$ & $87.9 \pm 5.7$ & $13.1 \pm 0.4$ & $81.0 \pm 5.6$ \\
\hline & After intervention & $12.7 \pm 0.4$ & $80.3 \pm 6.9$ & $13.8 \pm 0.7$ & $71.5 \pm 7.1$ \\
\hline & Ischemia 5 min & $0.0 \pm 0.0^{*}$ & $0.4 \pm 0.1^{*}$ & $0.0 \pm 0.0^{*}$ & $0.4 \pm 0.1^{*}$ \\
\hline & Ischemia $25 \mathrm{~min}$ & $0.0 \pm 0.0^{*}$ & $0.3 \pm 0.1^{*}$ & $0.0 \pm 0.0^{\star}$ & $0.2 \pm 0.1^{*}$ \\
\hline & Reperfusion 10 min & $8.0 \pm 0.7^{\star \dagger}$ & $30.1 \pm 5.7^{\star}$ & $8.8 \pm 0.6^{\star}$ & $34.6 \pm 5.2^{\star}$ \\
\hline & Reperfusion 30 min & $7.3 \pm 0.7^{* \dagger}$ & $34.1 \pm 5.8^{*}$ & - & - \\
\hline \multirow{6}{*}{$\begin{array}{l}\text { pRIPC } \\
(n=10 / 10)\end{array}$} & Baseline & $12.6 \pm 0.5$ & $88.7 \pm 8.2$ & $12.1 \pm 0.4$ & $91.8 \pm 3.3$ \\
\hline & After intervention & $12.8 \pm 0.9$ & $80.5 \pm 9.9$ & $13.3 \pm 0.4$ & $87.8 \pm 3.1$ \\
\hline & Ischemia 5 min & $0.0 \pm 0.0^{*}$ & $0.3 \pm 0.1^{*}$ & $0.0 \pm 0.0^{*}$ & $0.5 \pm 0.3^{*}$ \\
\hline & Ischemia $25 \mathrm{~min}$ & $0.0 \pm 0.0^{*}$ & $0.5 \pm 0.4^{*}$ & $0.0 \pm 0.0^{*}$ & $0.2 \pm 0.1^{*}$ \\
\hline & Reperfusion $10 \mathrm{~min}$ & $9.0 \pm 0.8^{\star \dagger}$ & $30.4 \pm 8.4^{*}$ & $8.9 \pm 0.8^{*}$ & $21.7 \pm 9.0^{*}$ \\
\hline & Reperfusion 30 min & $8.1 \pm 0.9^{* \dagger}$ & $28.8 \pm 8.4^{*}$ & - & - \\
\hline \multirow{6}{*}{$\begin{array}{l}\text { IPC } \\
(n=8 / 11)\end{array}$} & Baseline & $12.0 \pm 0.6$ & $79.8 \pm 7.8$ & $12.1 \pm 0.6$ & $95.0 \pm 5.4$ \\
\hline & After intervention & $12.2 \pm 0.5$ & $70.4 \pm 7.5$ & $12.4 \pm 0.6$ & $85.1 \pm 5.1$ \\
\hline & Ischemia 5 min & $0.0 \pm 0.0^{*}$ & $1.5 \pm 1.0^{*}$ & $0.0 \pm 0.0^{*}$ & $0.6 \pm 0.2^{*}$ \\
\hline & Ischemia $25 \mathrm{~min}$ & $0.0 \pm 0.0^{*}$ & $2.0 \pm 1.7^{\star}$ & $0.0 \pm 0.0^{\star}$ & $4.8 \pm 4.2^{*}$ \\
\hline & Reperfusion $10 \mathrm{~min}$ & $12.3 \pm 0.7^{\dagger}$ & $44.7 \pm 11.6^{\star \dagger}$ & $11.8 \pm 1.0^{\#}$ & $69.6 \pm 10.4^{* \dagger}$ \\
\hline & Reperfusion 30 min & $10.9 \pm 0.6^{\dagger}$ & $51.0 \pm 13.5^{\star \dagger}$ & - & - \\
\hline \multirow{6}{*}{$\begin{array}{l}\text { TNF- } \alpha \\
\text { infusion } \\
(n=7 / 8)\end{array}$} & Baseline & $12.8 \pm 0.7$ & $75.3 \pm 7.6$ & $13.6 \pm 0.6$ & $93.9 \pm 5.8$ \\
\hline & After intervention & $12.0 \pm 0.9$ & $70.5 \pm 9.0$ & $13.6 \pm 0.5$ & $96.6 \pm 5.8$ \\
\hline & Ischemia 5 min & $0.0 \pm 0.0^{*}$ & $0.6 \pm 0.1^{*}$ & $0.0 \pm 0.0^{*}$ & $0.6 \pm 0.1^{*}$ \\
\hline & Ischemia $25 \mathrm{~min}$ & $0.0 \pm 0.0^{*}$ & $0.1 \pm 0.0^{*}$ & $0.0 \pm 0.0^{*}$ & $0.4 \pm 0.2^{*}$ \\
\hline & Reperfusion 10 min & $7.4 \pm 0.9^{* \dagger}$ & $22.8 \pm 3.0^{*}$ & $9.7 \pm 1.1^{\star}$ & $34.3 \pm 6.1^{*}$ \\
\hline & Reperfusion $30 \mathrm{~min}$ & $6.9 \pm 0.8^{* \dagger}$ & $19.1 \pm 3.8^{\star}$ & - & - \\
\hline \multirow{7}{*}{$\begin{array}{l}\text { Sham } \\
(n=6 / 8)\end{array}$} & Analogue to & & & & \\
\hline & Baseline & $12.8 \pm 1.4$ & $84.9 \pm 9.1$ & $12.2 \pm 0.7$ & $95.1 \pm 3.2$ \\
\hline & After intervention & $12.7 \pm 1.3$ & $87.7 \pm 9.0$ & $12.0 \pm 0.7$ & $95.0 \pm 3.6$ \\
\hline & Ischemia 5 min & $12.4 \pm 1.3$ & $85.6 \pm 9.1$ & $11.8 \pm 0.7$ & $94.0 \pm 3.7$ \\
\hline & Ischemia $25 \mathrm{~min}$ & $11.9 \pm 1.3$ & $84.5 \pm 9.4$ & $11.3 \pm 0.9$ & $88.0 \pm 4.9$ \\
\hline & Reperfusion 10 min & $11.7 \pm 1.3$ & $83.3 \pm 8.5$ & $10.9 \pm 0.8$ & $83.7 \pm 4.4$ \\
\hline & Reperfusion 30 min & $11.5 \pm 1.1$ & $83.3 \pm 8.3$ & - & - \\
\hline
\end{tabular}

Mean coronary perfusate flow (CF $F_{\text {mean }}$ ) and maximal developed left ventricular pressure (LVDP $\left.P_{\max }\right)$ of isolated perfused rat hearts with ischemia/reperfusion $(I / R)$ and I/R with plasma taken from pigs after placebo (pPlacebo), with plasma taken from pigs after remote ischemic preconditioning (PRIPC), with local ischemic preconditioning (IPC), with tumor necrosis factor $\alpha$ (TNF- $\alpha$ ) infusion and without I/R (sham). $C F_{\text {mean }}$ and $L V D P_{\max }$ were analyzed at different time points: at baseline, after plasma infusion/IPC/TNF- $\alpha$ infusion (intervention), at 5/25 min ischemia, and at 10/30 min reperfusion. Mean \pm SEM; ${ }^{*} p<0.05 \mathrm{vs}$. baseline; ${ }^{\dagger} p<0.05 \mathrm{vs} .1 / R$. 


\section{Mitochondrial function}

ADP-stimulated complex I respiration was reduced after I/R compared to sham. With placebo pig plasma ADP-stimulated complex I respiration was not different from that with I/R. RIPC pig plasma improved ADP-stimulated complex I respiration over that with placebo pig plasma and I/R, as did IPC and TNF- $\alpha$ (Figure 2 A). Mitochondrial complex IV respiration and maximal oxygen uptake of uncoupled mitochondria were not different between groups, reflecting an equal loading of viable mitochondria (Figures $2 \mathrm{~B}$ ).

The mitochondrial ATP production was decreased after I/R compared to sham. Placebo pig plasma did not cause any difference to I/R. The RIPC pig plasma increased ATP production over that with placebo pig plasma and I/R. ATP production was increased with IPC and TNF $\alpha$ over that with I/R (Figure 3).

The CRC of mitochondria was reduced after I/R compared to sham. CRC with placebo pig plasma was comparable with that after I/R. RIPC pig plasma improved CRC over that with placebo pig plasma and I/R. Local IPC and TNF- $\alpha$ improved CRC over that with I/R (Figure $4 \mathrm{~A}$ ). With cyclosporine $A, C R C$ was not different between groups (Figure $4 \mathrm{~B}$ ).

Mitochondrial ROS production after I/R was higher compared to sham. The ROS production with placebo pig plasma was comparable with that in I/R. RIPC pig plasma reduced the ROS production. Local IPC and TNF- $\alpha$ also reduced the ROS production (Figure 5).

\section{Discussion}

In the present study, improved mitochondrial function at early reperfusion was associated with cardioprotection by humoral transfer of RIPC pig plasma to isolated perfused rat hearts, supporting

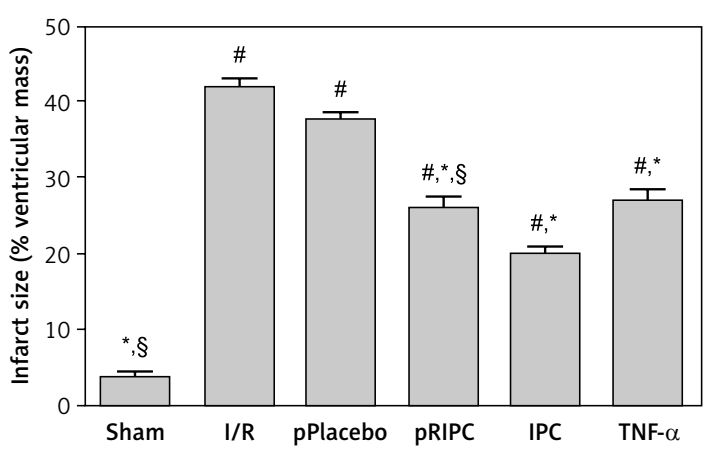

Figure 1. Infarct size in isolated rat hearts after sham $(n=6)$, ischemia/reperfusion (I/R; $n=8)$ and I/R with plasma taken from pigs after placebo (pPlacebo; $n=10$ ), with plasma taken from pigs after remote ischemic preconditioning ( $\mathrm{PRIPC} ; n=10$ ), with local ischemic preconditioning (IPC; $n=8)$ and with tumor necrosis factor $\alpha$ (TNF- $\alpha ; n=7)$ infusion ${ }^{\#} P<0.05$ vs. sham, ${ }^{*} p<0.05$ vs. $1 / R,{ }^{\S} p<0.05$ vs. placebo.

the notion that mitochondria are a myocardial target organelle of RIPC's protection.

The extent of infarct size reduction and the improvement of mitochondrial function by RIPC pig plasma were comparable to those induced by local IPC or TNF- $\alpha$ infusion, whereas the recovery of rat heart LVDP during reperfusion was better with local IPC than in response to RIPC pig plasma or TNF- $\alpha$. These findings suggest that improved mitochondrial function plays no important role in the functional recovery of the rat heart, except for the reduction of infarct size. The IPC is a most effective local ischemic conditioning maneuver and is associated with improved mitochondrial function [40, 41]. The cytokine TNF- $\alpha$ activates intracellular cardioprotective signaling [42, 43], which converges at the mitochondria [21]. The TNF- $\alpha$ has an ambivalent role in myocardial ischemia/ reperfusion injury and during myocardial protection [32]. Both IPC [44-46] and remote ischemic postconditioning [47] depress the I/R-induced in-
A

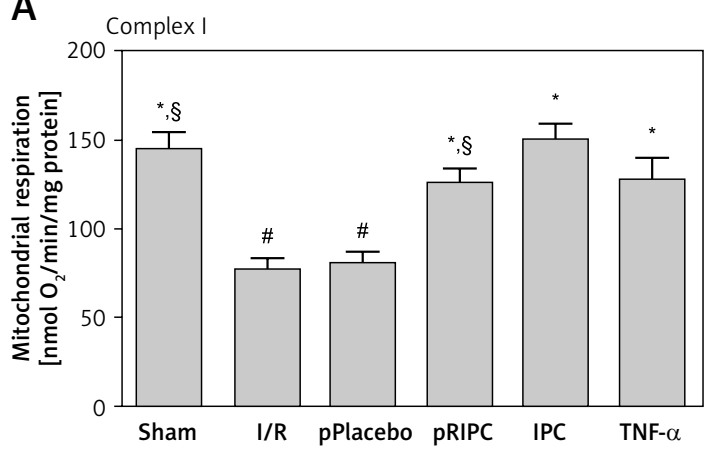

B

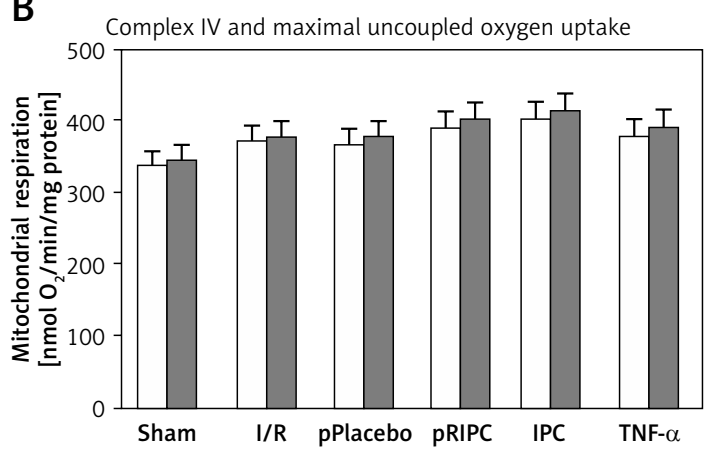

Figure 2. Respiration of isolated mitochondria from rat hearts after sham $(n=8)$, ischemia/reperfusion $(I / R ; n=8)$ and I/R with plasma taken from pigs after placebo (pPlacebo; $n=10$ ), with plasma taken from pigs after remote ischemic preconditioning ( $P$ RIPC; $n=10$ ), with local ischemic preconditioning (IPC; $n=11$ ) and with tumor necrosis factor $\alpha$ (TNF- $\alpha ; n=8)$ infusion. A - Adenosine diphosphate (ADP)-stimulated complex I respiration. B - Complex IV respiration with TMPD and ascorbate (white) and maximal uncoupled oxygen uptake with FCCP (grey)

${ }^{\#} P<0.05$ vs. sham, ${ }^{*} p<0.05$ vs. $1 / R,{ }^{\S} p<0.05$ vs. placebo. 


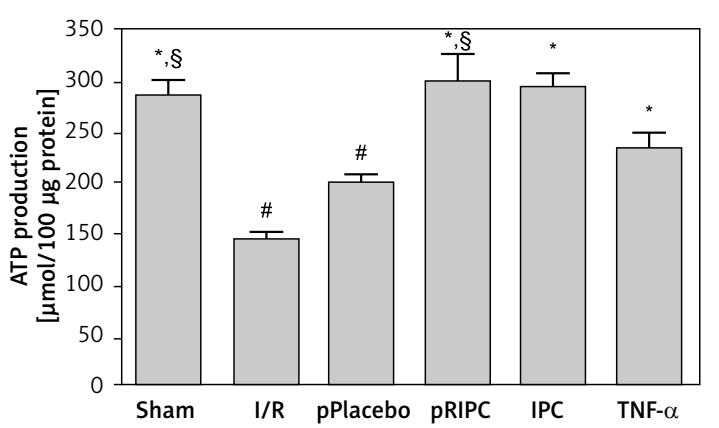

Figure 3. Adenosine triphosphate (ATP) production of isolated mitochondria from rat hearts after sham $(n=8)$, ischemia/reperfusion (I/R; $n=8)$ and I/R with plasma taken from pigs after placebo (pPlacebo; $n=8$ ), with plasma taken from pigs after remote ischemic preconditioning ( $p$ RIPC; $n=8$ ), with local ischemic preconditioning (IPC; $n=11$ ) and with tumor necrosis factor $\alpha$ (TNF- $\alpha ; n=8)$ infusion

${ }^{\#} P<0.05$ vs. sham, ${ }^{*} p<0.05$ vs. $I / R,{ }^{\S} p<0.05$ vs. placebo.

crease of endogenous TNF- $\alpha$ in rodents. On the other hand, exogenous TNF- $\alpha$ mimics IPC cardioprotection when administered prior to ischemia [48], and protection by IPC is lost in TNF- $\alpha-K O$ mice [49]. Cardioprotection by exogenous TNF- $\alpha$ is dose-dependent: whereas lower doses are protective, higher TNF- $\alpha$ doses even increase infarct size [34]. Thus, TNF- $\alpha$ has bidirectional effects: TNF- $\alpha$ at low concentrations acts as a signaling molecule, but TNF- $\alpha$ at higher concentrations induces irreversible cell damage. The protection by TNF- $\alpha$ is also associated with restoration of mitochondrial respiration [48] and inhibition of MPTP opening [50].

Prior studies suggested that RIPC is also associated with improved mitochondrial respiration [25-28] but did not look for an association with infarct size reduction. Here, we confirmed improved respiration of mitochondria isolated from rat hearts after RIPC pig plasma transfer, and cardioprotection by RIPC was evidenced in the RIPC

A

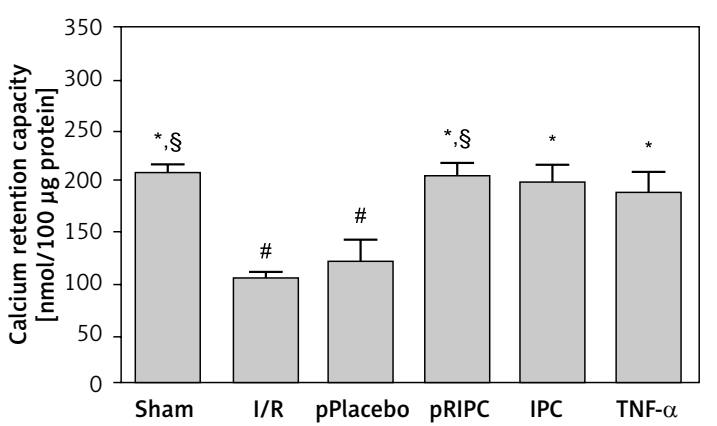

plasma donor pigs as well as in the plasma acceptor rat hearts by infarct size reduction.

A logical consequence of increased ADP-stimulated respiration is increased ATP production [51]. Exactly such increased ATP production was evident in parallel to the increased respiration by RIPC pig plasma, local IPC and TNF- $\alpha$ infusion.

Improved mitochondrial respiration after local ischemic postconditioning in anesthetized pig hearts was also associated with increased CRC of mitochondria isolated at 10 min reperfusion [52]. As in our previous study on ischemic postconditioning, the improved mitochondrial respiration was associated with an increased CRC in the present study, indicating inhibition of mPTP opening. Opening of the MPTP induces cell death; therefore the inhibition of MPTP opening is decisive for cardiomyocyte survival at early reperfusion $[29-31,53]$. Inhibition of MPTP opening by cyclosporine $A$ attenuates reperfusion injury in animals [54, 55], as it also did in a small cohort of patients with acute myocardial infarction [56]. However, this proof-of-concept study was not confirmed by two recent large-scaled randomized trials, CIRCUS and CYCLE, where cyclosporine A neither reduced biomarker release nor improved clinical outcome of patients with acute myocardial infarction [57, 58]. The reasons for the discrepancy between the proofof-concept and the two phase III trials are not clear in detail, but may include different patient inclusion criteria, notably an increased time from symptom onset to reperfusion, greater pre-existing protection by more widespread use of novel $P_{2 Y_{12}}$ antagonists, a different vehicle used to disolve cyclosporine $A$ in CIRCUS but not in CYCLE, inadequate delivery of the drug to the myocardium at early reperfusion, and a potential false-positive type I error [59].

In contrast to our study, humoral transfer of RIPC rabbit plasma dialysate to isolated neonatal rabbit hearts did not affect the MPTP opening of mitochondria isolated after $30 \mathrm{~min}$ reperfusion [28]. The opening of mPTP could have been

B

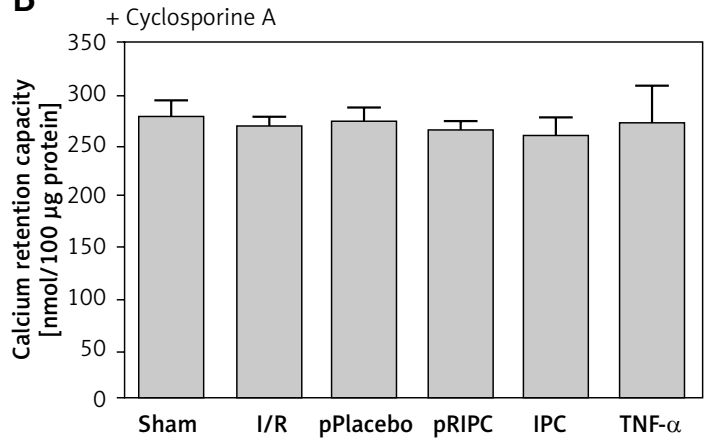

Figure 4. Calcium retention capacity of isolated mitochondria from rat hearts after sham $(n=8)$, ischemia/reperfusion (I/R; $n=8)$ and I/R with plasma taken from pigs after placebo (pPlacebo; $n=8$ ), with plasma taken from pigs after remote ischemic preconditioning (pRIPC; $n=8$ ), with local ischemic preconditioning (IPC; $n=8)$ and with tumor necrosis factor $\alpha$ (TNF- $\alpha ; n=8)$ infusion (A). Positive controls with addition of cyclosporine A (B)

${ }^{\#} P<0.05$ vs. sham, ${ }^{*} p<0.05$ vs. $I / R,{ }^{8} p<0.05$ vs. placebo. 
missed due to a late observation time point during reperfusion [60] or by absence of cardioprotection, which was not assured [28].

In an in vivo murine model with RIPC cardioprotection, S-nitrosation of mitochondrial complex I reduced its activity and finally reduced myocardial ROS production [19]. Here, the transfer of RIPC pig plasma attenuated I/R induced mitochondrial ROS production after improved ADP-stimulated complex I respiration, indicating that mitochondria are involved in the reduction of ROS formation.

The RIPC-induced factor(s) which may contribute to modulation and preservation of mitochondrial function have not been identified yet. In an isolated neonatal rabbit heart model, remote cardioprotection by IPC effluent (from isolated rabbit hearts) preserved mitochondrial integrity and function in an adenosine receptor dependent mechanism. Although infarct size reduction could be mimicked by adenosine, IPC effluent contained additional factor(s) contributing to modulation of the mitochondrial response to I/R injury [61]. In our model, RIPC activated the survival activating factor enhancement (SAFE) pathway, notably signal transducer and activator of transcription 3 (STAT3) in the pig myocardium and both the reperfusion injury salvage kinase (RISK) and the SAFE pathway in the rat myocardium [18]. The activation of mitochondrial STAT3 was causally involved in infarct size reduction by ischemic postconditioning in pigs and associated with preservation of mitochondrial complex I respiration and CRC [52]. Whether RIPC activates mitochondrial STAT3 in association with improved mitochondrial function requires further investigation.

In this study we demonstrated improved mitochondrial function associated with infarct size reduction in isolated perfused rat hearts after humoral transfer of RIPC. However, a causal relationship between preserved mitochondrial function by RIPC and infarct size reduction remains to be established.

The extent of cardioprotection and improvement of mitochondrial function by RIPC pig plasma was comparable to that induced by local IPC. However, other algorithms for IPC and for RIPC but also changes in the concentration of humoral factor transfer to the isolated rat heart may influence the extent of protection. Placebo pig plasma tended to reduce infarct size and increase mitochondrial ATP production compared to the values observed in hearts without plasma infusion. This slight protection could be caused by the plasma protein content itself, e.g. in isolated perfused rat hearts, trace amounts (0.0004\%) of bovine or rat serum albumin protected against I/R injury [62]. Also, plasma derived hormones [63] or cytokines [43] can induce cardioprotection. Species-specific differences in hormone or cytokine levels be-

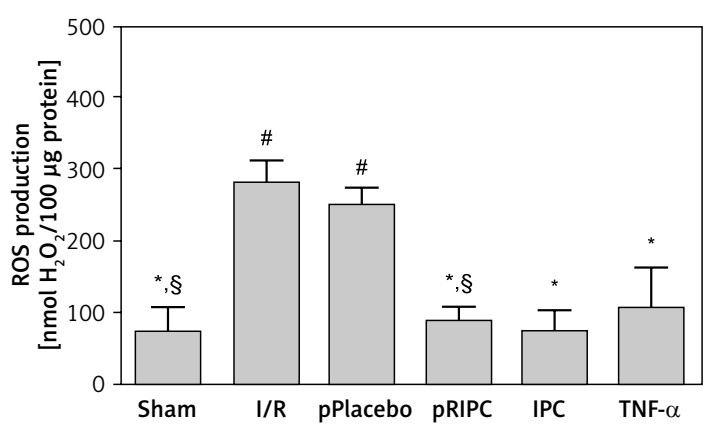

Figure 5. Reactive oxygen species (ROS) production of isolated mitochondria from rat hearts after sham $(n=6)$, ischemia/reperfusion (I/R; $n=7)$ and I/R with plasma taken from pigs after placebo (pPlacebo; $n=8$ ), with plasma taken from pigs after remote ischemic preconditioning (pRIPC; $n=7$ ), with local ischemic preconditioning (IPC; $n=7$ ) and with tumor necrosis factor $\alpha$ (TNF- $\alpha ; n=7$ ) infusion

${ }^{\#} P<0.05$ vs. sham, ${ }^{*} p<0.05$ vs. $I / R,{ }^{s} p<0.05$ vs. placebo.

tween pig and rat plasma may further contribute to protection; e.g. in pig plasma/serum, the concentration of the cytokine TNF- $\alpha$ is higher (50$150 \mathrm{pg} / \mathrm{ml})$ [64-66] than in rat plasma ( 10 pg/ $\mathrm{ml})[67,68]$.

Mitochondrial function was measured at one time point of early reperfusion, and these results are not transferable to the whole reperfusion period [69]. However, the first minutes of reperfusion after the sustained ischemia have been emphasized as critical for signal transduction of cardioprotection and mitochondrial function $[22,69]$. Mitochondrial function was assessed using a mixture of mitochondrial fractions, the subsarcolemmal and the interfibrillar mitochondria. Prior studies used subsarcolemmal mitochondria $[19,28]$ or saponin-permeabilized cardiac fibers [26, 27]. Subsarcolemmal mitochondria are more vulnerable to ischemic injury and to mitochondrial calcium overload than interfibrillar mitochondria [70, 71].

All measurements of mitochondrial function were made at physiological temperature of $37^{\circ} \mathrm{C}$. At this temperature, respiration rates are increased and may not be comparable to mitochondrial respiration measured at $25^{\circ} \mathrm{C}$ [72].

In conclusion, the present study provides evidence that myocardial protection by across-species transfer of RIPC pig plasma to isolated perfused rat hearts is associated with improved mitochondrial function at early reperfusion, supporting the notion that mitochondria are an intracellular target of protection by RIPC.

\section{Acknowledgments}

Nilguen Gedik and Leonardo Maciel contributed equally to this work.

Grants - German Research Foundation (SFB 1116 B8). 


\section{Conflict of interest}

The authors declare no conflict of interest.

\section{References}

1. Murry CE, Jennings RB, Reimer KA. Preconditioning with ischemia: a delay of lethal cell injury in ischemic myocardium. Circulation 1986; 74: 1124-36.

2. Heusch G, Botker HE, Przyklenk K, Redington A, Yellon DM. Remote ischemic conditioning. J Am Coll Cardiol 2015; 65: 177-95.

3. Davies WR, Brown AJ, Watson W, et al. Remote ischemic preconditioning improves outcome at 6 years after elective percutaneous coronary intervention: the CRISP stent trial long-term follow-up. Circ Cardiovasc Interv 2013; 6: 246-51.

4. Hausenloy DJ, Mwamure PK, Venugopal V, et al. Effect of remote ischaemic preconditioning on myocardial injury in patients undergoing coronary artery bypass graft surgery: a randomized controlled trial. Lancet 2007; 370: 575-9.

5. Thielmann M, Kottenberg E, Kleinbongard P, et al. Cardioprotective and prognostic effects of remote ischaemic preconditioning in patients undergoing coronary artery bypass surgery: a single-centre randomised, double-blind, controlled trial. Lancet 2013; 382: 597-604.

6. Candilio L, Malik A, Ariti C, et al. Effect of remote ischaemic preconditioning on clinical outcomes in patients undergoing cardiac bypass surgery: a randomised controlled clinical trial. Heart 2015; 10: 185-92.

7. Bøtker HE, Kharbanda R, Schmidt MR, et al. Remote ischaemic conditioning before hospital admission, as a complement to angioplasty, and effect on myocardial salvage in patients with acute myocardial infarction: a randomised trial. Lancet 2010; 375: 727-34.

8. Eitel I, Stiermaier T, Rommel KP, et al. Cardioprotection by combined intrahospital remote ischaemic perconditioning and postconditioning in ST-elevation myocardial infarction: the randomized LIPSIA CONDITIONING trial. Eur Heart J 2015; 36: 3049-57.

9. Yellon DM, Ackbarkhan AK, Balgobin V, et al. Remote ischemic conditioning reduces myocardial infarct size in STEMI patients treated by thrombolysis. J Am Coll Cardiol 2015; 65: 2764-5.

10. White SK, Frohlich GM, Sado DM, et al. Remote ischemic conditioning reduces myocardial infarct size and edema in patients with ST-segment elevation myocardial infarction. J Am Coll Cardiol Cardiovasc Interv 2015; 8: 178-88.

11. Sloth AD, Schmidt MR, Munk K, et al. Improved longterm clinical outcomes in patients with ST-elevation myocardial infarction undergoing remote ischaemic conditioning as an adjunct to primary percutaneous coronary intervention. Eur Heart J 2014; 35: 168-75.

12. Hausenloy DJ, Candilio L, Evans R, et al. Remote ischemic preconditioning and outcomes of cardiac surgery. N Engl J Med 2015; 373: 1408-17.

13. Meybohm P, Bein B, Brosteanu O, et al. A multicenter trial of remote ischemic preconditioning for heart surgery. N Engl J Med 2015; 373: 1397-407.

14. Heusch G, Gersh BJ. ERICCA and RIPHeart: two nails in the coffin for cardioprotection by remote ischemic conditioning? Probably not! Eur Heart J 2015; 37: 200-1.

15. Heusch G, Gersh BJ. The pathophysiology of acute myocardial infarction and strategies of protection beyond reperfusion: a continual challenge. Eur Heart J 2016; doi:10.1093/eurheartj/ehw224.
16. Shimizu M, Tropak M, Diaz RJ, et al. Transient limb ischaemia remotely preconditions through a humoral mechanism acting directly on the myocardium: evidence suggesting cross-species protection. Clin Sci (Lond) 2009; 117: 191-200.

17. Dickson EW, Lorbar M, Porcaro WA, et al. Rabbit heart can be "preconditioned" via transfer of coronary effluent. Am J Physiol 1999; 277: H2451-7.

18. Skyschally A, Gent S, Amanakis G, Schulte C, Kleinbongard P, Heusch G. Across-species transfer of protection by remote ischemic preconditioning with species-specific myocardial signal transduction by reperfusion injury salvage kinase and survival activating factor enhancement pathways. Circ Res 2015; 117: 279-88.

19. Rassaf T, Totzeck M, Hendgen-Cotta UB, Shiva S, Heusch G, Kelm M. Circulating nitrite contributes to cardioprotection by remote ischemic preconditioning. Circ Res 2014; 114: 1601-10.

20. Jensen RV, Stottrup NB, Kristiansen SB, Bøtker HE. Release of a humoral circulating cardioprotective factor by remote ischemic preconditioning is dependent on preserved neural pathways in diabetic patients. Basic Res Cardiol 2012; 107: 285.

21. Heusch G, Boengler K, Schulz R. Cardioprotection: nitric oxide, protein kinases, and mitochondria. Circulation 2008; 118: 1915-9.

22. Heusch G. Molecular basis of cardioprotection: signal transduction in ischemic pre-, post- and remote conditioning. Circ Res 2015; 116: 674-99.

23. Cellier L, Tamareille S, Kalakech H, et al. Remote ischemic conditioning influences mitochondrial dynamics. Shock 2016; 45: 192-7.

24. Cao Y, Zhang SZ, Zhao SQ, Bruce IC. The mitochondrial $\mathrm{Ca}(2+)$-activated $\mathrm{K}(+)$ channel contributes to cardioprotection by limb remote ischemic preconditioning in rat. Life Sci 2011; 88: 1026-30.

25. Ferko $M$, Kancirova I, Jasova $M$, et al. Remote ischemic preconditioning of the heart: protective responses in functional and biophysical properties of cardiac mitochondria. Physiol Res 2014; 63 Suppl 4: S469-78.

26. Slagsvold KH, Moreira JB, Rognmo O, et al. Remote ischemic preconditioning preserves mitochondrial function and activates pro-survival protein kinase Akt in the left ventricle during cardiac surgery: a randomized trial. Int J Cardiol 2014; 177: 409-17.

27. Slagsvold KH, Rognmo O, Hoydal M, Wisloff U, Wahba A. Remote ischemic preconditioning preserves mitochondrial function and influences myocardial microRNA expression in atrial myocardium during coronary bypass surgery. Circ Res 2014; 114: 851-9.

28. Wang L, Oka N, Tropak M, et al. Remote ischemic preconditioning elaborates a transferable blood-borne effector that protects mitochondrial structure and function and preserves myocardial performance after neonatal cardioplegic arrest. J Thorac Cardiovasc Surg 2008; 136: 335-42.

29. Heusch G, Boengler K, Schulz R. Inhibition of mitochondrial permeability transition pore opening: the holy grail of cardioprotection. Basic Res Cardiol 2010; 105: 151-4.

30. Griffiths EJ, Halestrap AP. Mitochondrial non-specific pores remain closed during cardiac ischaemia, but open upon reperfusion. Biochem J 1995; 307: 93-8.

31. Cohen MV, Yang XM, Downey JM. Acidosis, oxygen, and interference with mitochondrial permeability transition pore formation in the early minutes of reperfusion are critical to postconditioning's success. Basic Res Cardiol 2008; 103: 464-71. 
32. Skyschally A, Gres P, Hoffmann S, et al. Bidirectional role of tumor necrosis factor-alpha in coronary microembolization: progressive contractile dysfunction versus delayed protection against infarction. Circ Res 2007; 100: 140-6.

33. Heusch P, Skyschally A, Leineweber K, Haude M, Erbel R, Heusch G. The interaction of coronary microembolization and ischemic preconditioning: a third window of cardioprotection through TNF-alpha. Arch Med Sci 2007; 3: 83-92.

34. Kleinbongard P, Schulz R, Heusch G. TNFalpha in myocardial ischemia/reperfusion, remodeling and heart failure. Heart FailRev 2011; 16: 49-69.

35. Bayne K. Revised guide for the care and use of laboratory animals available. American Physiological Society. Physiologist 1996; 39: 199, 208-11.

36. Lecour S, Smith RM, Woodward B, Opie LH, Rochette L, Sack MN. Identification of a novel role for sphingolipid signaling in TNFalpha and ischemic preconditioning mediated cardioprotection. J Mol Cell Cardiol 2002; 34 509-18.

37. Kleinbongard P, Gedik N, Witting P, Freedman B, Klocker N, Heusch G. Pleiotropic, heart rate-independent cardioprotection by ivabradine. Br J Pharmacol 2015; 172: 4380-90

38. Gedik N, Heusch G, Skyschally A. Infarct size reduction by cyclosporine $A$ at reperfusion involves inhibition of the mitochondrial permeability transition pore but does not improve mitochondrial respiration. Arch Med Sci 2013; 9: 968-75.

39. Basso E, Fante L, Fowlkes J, Petronilli V, Forte MA, Bernardi P. Properties of the permeability transition pore in mitochondria devoid of cyclophilin D. J Biol Chem 2005; 280: 18558-61.

40. Argaud L, Gateau-Roesch O, Chalabreysse L, et al. Preconditioning delays $\mathrm{Ca} 2+$-induced mitochondrial permeability transition. Cardiovasc Res 2004; 61: 115-22.

41. Halestrap AP, Clarke SJ, Khaliulin I. The role of mitochondria in protection of the heart by preconditioning. Biochim Biophys Acta 2007; 1767: 1007-31.

42. Lacerda L, Somers S, Opie LH, Lecour S. Ischemic postconditioning protects against reperfusion injury via the SAFE pathway. Cardiovasc Res 2009; 84: 201-8.

43. Lecour S, James RW. When are pro-inflammatory cytokines SAFE in heart failure? Eur Heart J 2011; 32: 680-5.

44. Kimura H, Shintani-Ishida K, Nakajima M, Liu S, Matsumoto $\mathrm{K}$, Yoshida $\mathrm{K}$. Ischemic preconditioning or p38 MAP kinase inhibition attenuates myocardial TNF-alpha production and mitochondria damage in brief myocardial ischemia. Life Sci 2006; 78: 1901-10.

45. Meldrum DR, Dinarello CA, Shames BD, et al. Ischemic preconditioning decreases postischemic myocardial tumor necrosis factor-alpha production. Potential ultimate effector mechanism of preconditioning. Circulation 1998; 98: II-214-9.

46. Belosjorow S, Schulz R, Dörge H, Schade FU, Heusch G. Endotoxin and ischemic preconditioning: TNF-alpha concentration and myocardial infarct development in rabbits. Am J Physiol Heart Circ Physiol 1999; 277: H2470-5.

47. Zhang JQ, Wang Q, Xue FS, et al. Ischemic preconditioning produces more powerful anti-inflammatory and cardioprotective effects than limb remote ischemic postconditioning in rats with myocardial ischemia-reperfusion injury. Chin Med J (Engl) 2013; 126: 3949-55.

48. Lecour S, Suleman N, Deuchar GA, et al. Pharmacological preconditioning with tumor necrosis factor-alpha activates signal transducer and activator of transcription-3 at reperfusion without involving classic prosurvival kinases (Akt and extracellular signal-regulated kinase). Circulation 2005; 112: 3911-8.

49. Smith RM, Suleman N, McCarthy J, Sack MN. Classic ischemic but not pharmacologic preconditioning is abrogated following genetic ablation of the TNF-alpha gene. Cardiovasc Res 2002; 55: 553-60.

50. Gao Q, Zhang SZ, Cao CM, Bruce IC, Xia Q. The mitochondrial permeability transition pore and the $\mathrm{Ca} 2+$-activated $\mathrm{K}+$ channel contribute to the cardioprotection conferred by tumor necrosis factor-alpha. Cytokine 2005; 32: 199-205.

51. Doenst T, Nguyen TD, Abel ED. Cardiac metabolism in heart failure: implications beyond ATP production. Circ Res 2013; 113: 709-24.

52. Heusch G, Musiolik J, Gedik N, Skyschally A. Mitochondrial STAT3 activation and cardioprotection by ischemic postconditioning in pigs with regional myocardial ischemia/reperfusion. Circ Res 2011; 109: 1302-8.

53. Yang S, Li H, Tang L, et al. Apelin-13 protects the heart against ischemia-reperfusion injury through the RISK-GSK-3beta-mPTP pathway. Arch Med Sci 2015; 11 : 1065-73.

54. Skyschally A, Schulz R, Heusch G. Cyclosporine A at reperfusion reduces infarct size in pigs. Cardiovasc Drugs Ther 2010; 24: 85-7.

55. Argaud L, Gateau-Roesch O, Muntean D, et al. Specific inhibition of the mitochondrial permeability transition prevents lethal reperfusion injury. J Mol Cell Cardiol 2005; 38: 367-74.

56. Piot C, Croisille P, Staat P, et al. Effect of cyclosporine on reperfusion injury in acute myocardial infarction. New Engl J Med 2008; 359: 473-81.

57. Cung TT, Morel O, Cayla G, et al. Cyclosporine before $\mathrm{PCl}$ in patients with acute myocardial infarction. $N$ Engl J Med 2015; 373: 1021-103.

58. Ottani F, Latini R, Staszewsky L, et al. Cyclosporine A in reperfused myocardial infarction. The multicenter, contolled, open-label CYCLE Trial. J Am Coll Cardiol 2016; 67: 365-74.

59. Heusch G. CIRCUS - a kiss of death for cardioprotection? Cardiovasc Res 2015; 108: 215-6.

60. Kerr PM, Suleiman MS, Halestrap AP. Reversal of permeability transition during recovery of hearts from ischemia and its enhancement by pyruvate. Am J Physiol Heart Circ Physiol 1999; 276: H496-502.

61. Leung CH, Wang L, Nielsen JM, et al. Remote cardioprotection by transfer of coronary effluent from ischemic preconditioned rabbit heart preserves mitochondrial integrity and function via adenosine receptor activation. Cardiovasc Drugs Ther 2014; 28: 7-17.

62. Watts JA, Maiorano PC. Trace amounts of albumin protect against ischemia and reperfusion injury in isolated rat hearts. J Mol Cell Cardiol 1999; 31: 1653-62.

63. Burley DS, Hamid SA, Baxter GF. Cardioprotective actions of peptide hormones in myocardial ischemia. Heart FailRev 2007; 12: 279-91.

64. Kruse R, Essen-Gustavsson B, Fossum C, Jensen-Waern M. Blood concentrations of the cytokines IL-1beta, IL-6, IL-10, TNF-alpha and IFN-gamma during experimentally induced swine dysentery. Acta Vet Scand 2008; 50: 32.

65. Webel DM, Finck BN, Baker DH, Johnson RW. Time course of increased plasma cytokines, cortisol, and urea nitrogen in pigs following intraperitoneal injection of lipopolysaccharide. J Animal Sci 1997; 75: 1514-20. 
66. Xi S, Yin W, Wang Z, et al. A minipig model of high-fat/ high-sucrose diet-induced diabetes and atherosclerosis. Int J Exp Pathol 2004; 85: 223-31.

67. Ohta M, Tarnawski AS, Itani R, et al. Tumor necrosis factor alpha regulates nitric oxide synthase expression in portal hypertensive gastric mucosa of rats. Hepatology 1998; 27: 906-13.

68. Darwish SF, El-Bakly WM, Arafa HM, El-Demerdash E. Targeting TNF-alpha and NF-kappaB activation by bee venom: role in suppressing adjuvant induced arthritis and methotrexate hepatotoxicity in rats. PLoS One 2013; 8: e79284.

69. Bosetti F, Baracca A, Lenaz G, Solaini G. Increased state 4 mitochondrial respiration and swelling in early post-ischemic reperfusion of rat heart. FEBS Lett 2004; 563: 161-4.

70. Lesnefsky EJ, Slabe TJ, Stoll MS, Minkler PE, Hoppel CL. Myocardial ischemia selectively depletes cardiolipin in rabbit heart subsarcolemmal mitochondria. Am J Physiol Heart Circ Physiol 2001; 280: H2770-8.

71. Holmuhamedov EL, Oberlin A, Short K, Terzic A, Jahangir A. Cardiac subsarcolemmal and interfibrillar mitochondria display distinct responsiveness to protection by diazoxide. PLoS One 2012; 7: e44667.

72. Boengler K, Hilfiker-Kleiner D, Heusch G, Schulz R. Inhibition of permeability transition pore opening by mitochondrial STAT3 and its role in myocardial ischemia/ reperfusion. Basic Res Cardiol 2010; 105: 771-85. 